\title{
Effects of interband phototunneling and filling the bands in electroreflectance spectra of germanium
}

\author{
P.A. Gentsar, L.A. Matveeva, A.A. Kudryavtsev, E.F. Venger \\ Institute of Semiconductor Physics, NAS of Ukraine, 41 prospect Nauky, 03028 Kyiv, Ukraine \\ Phone: +38 (044) 2658338
}

\begin{abstract}
Electroreflectance spectra of chemically etched (110) surface of intrinsic germanium are measured in the range of $E_{1}, E_{1}+\Delta_{1}$ transitions for $\hat{e} \|[100]$ and $\hat{e} \|[110]$ directions of the polarization vector. Separated are isotropic (surface) and anisotropic (bulk) electroreflectance components. The spectrum of the bulk electroreflectance component (the Keldysh-Franz effect), as it can be deduced from its phase, corresponds to the case when energy bands are bent up if going to the surface. This situation is possible in the conditions of filling the bands only at presence of an extremum in the semiconductor energy scheme. Appearance of this extremum can be related to the fact that the electron wave function at the surface is equal to zero or defermined by mirror image force action.
\end{abstract}

Keywords: electroreflection, Keldysh-Franz effect, band filling, mirror image force.

Paper received 26.02.03; accepted for publication 16.06.03.

\section{Introduction}

Modulation spectroscopy spectra contain valuable information concerning properties of phase interfaces [13]. Especially actual here is the task of separation of surface and bulk contributions into electroreflection (ER) signals. This separation is necessary, for instance, when studying electrooptical effects during band filling [4], subsurface carrier movement quantization [5, 6], subsurface layer amorphization, multilayer systems, etc. In [7], the surface contribution was separated against the quantization effect by distinguishing between field dependences of this effect and the Keldysh-Franz one (KFE). The method to separate surface and bulk contributions into ER signal was also suggested in [8], where it was based on the analysis of electrooptical effect polarisation (tensor) anisotropy.

In the case, separated was some subsurface layer with the complex refraction index $N_{s}=n_{s}+i x_{s}$ that differs from the bulk one $N_{b}=n_{b}+i x_{b}$. The validity of this method was demonstrated analyzing ER spectra of (110) $n$-Ge sampes. In [9], applicability of this method was shown using analysis of ER spectra taken from $n$ - and $p$-type Ge samples after mechanical polishing their surface. A microwave-optical spectroscope that enables to study spectra of modulated light reflection in various layers of a semiconductor structure was offered and realized in [10].
Authors of $[8,11]$ noted that, in the case of intrinsic germanium $(i-\mathrm{Ge})$, the electromodulation spectrum of KFE bulk component in the range of $E_{1}, E_{1}+\Delta_{1}$ transitions, as to the form and alternation order of dominating extrema, corresponds to the situation when energy bands are bent up in the direction towards surface. This situation is possible in the band filling effect (BFE) conditions only at the presence of an extremum in the semiconductor energy band scheme in dependence on $x$ coordinate. The main purpose of this work is the investigate polarized ER spectra measured was to case of etched $i$-Ge surface to separate surface and bulk components of the signal and ascertain reasons of extremum appearance in the energy band scheme.

\section{Experimental}

ER spectra were measured taking Ge (110) samples with the impurity concentration close to the intrinsic one. In the range of transitions $E_{1}$ and $E_{1}+\Delta_{1}(2.0 \ldots 2.5 \mathrm{eV})$ we used the electrolytical method [1] at the frequency of the first harmonics $(2.2 \mathrm{kHz})$ at the room temperature in polarized light. Investigations were performed using potentials that correspond to minimal rates of electrochemical reactions at Ge-electrolyte interface. As an electrolyte we used the buffer solution of the following composition: $0.1 \mathrm{M} \mathrm{K}_{2} \mathrm{SO}_{4}, 0.025 \mathrm{M} \mathrm{Na}_{2} \mathrm{HPO}_{4}, 0.025 \mathrm{M}$ 
$\mathrm{KH}_{2} \mathrm{PO}_{4}$. Inaccuracy of determining the polarization anisotropy coefficient did not exceed $2 \%$.

\section{Results and discussion}

Depicted in Fig. 1 are ER spectra obtained in the case of etched i-Ge surface for two main directions of the polarization vector $\hat{e} \|[100]$ and $\hat{e} \|[\overline{1} 10]$. When processing these spectra, we used the procedure of separation of surface and bulk ER components described in [8].

Let us write ER signals measured in conditions of superposition of phototunneling and band filling effects for two different light polarizations $\hat{e} \|[\overline{1} 10]$

$$
\left(\frac{\Delta R}{R}\right)^{I}=\left(\frac{\Delta R}{R}\right)_{B F E}^{I}+\left(\frac{\Delta R}{R}\right)_{K F E}^{I}
$$

and $\hat{e} \|[100]$

$$
\left(\frac{\Delta R}{R}\right)^{I I}=\left(\frac{\Delta R}{R}\right)_{B F E}^{I I}+\left(\frac{\Delta R}{R}\right)_{K F E}^{I I}
$$

Taking into account polarization isotropy of BFE and anisotropy of KFE in applied measurement geometry, one can write

$$
\begin{aligned}
& \left(\frac{\Delta R}{R}\right)^{I}=\left(\frac{\Delta R}{R}\right)_{B F E}+\left(\frac{\Delta R}{R}\right)_{K F E}^{I} \\
& S_{1}\left(\frac{\Delta R}{R}\right)^{I}=\left(\frac{\Delta R}{R}\right)_{B F E}+S_{0}\left(\frac{\Delta R}{R}\right)_{K F E}^{I}
\end{aligned}
$$

It follows from (3) and (4) that

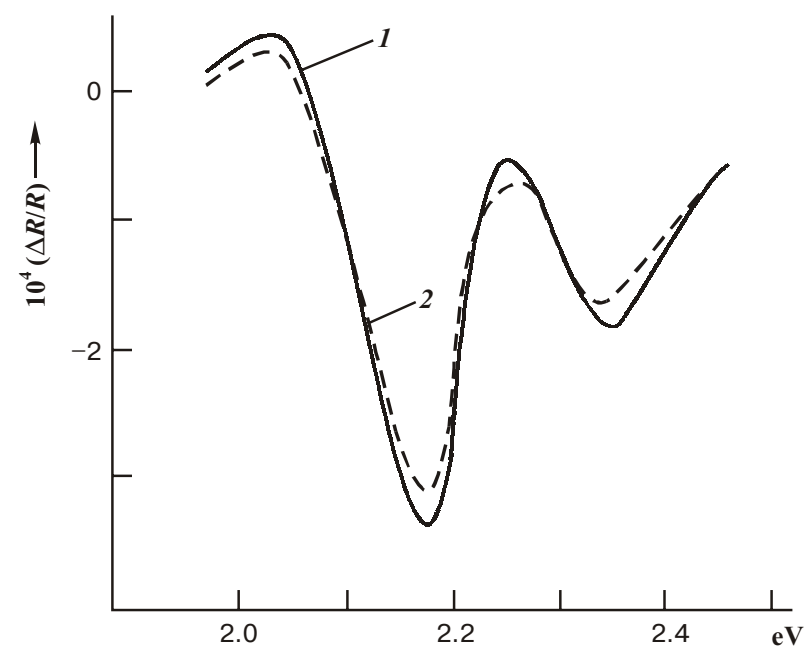

Fig. 1. $E R$ spectra for light polarizations $\hat{e} \|[100]$ and $\hat{e} \|[\overline{1} 10]$ in the case of the chemically etched (110) surface of intrinsic Ge, the shift relatively to a saturated calomel electrode being $-0.2 \mathrm{~V}$ and modulation magnitude $0.2 \mathrm{mV}$.

$$
\left(\frac{\Delta R}{R}\right)_{B F E}=\frac{S_{0}-S_{1}}{S_{0}-1} \frac{\Delta R^{I}}{R}
$$

$$
\left(\frac{\Delta R}{R}\right)_{K F E}=\frac{S_{1}-1}{S_{0}-1} \frac{\Delta R^{I}}{R}
$$

where $S_{1}$ and $S_{0}$ are polarization anisotropy coefficients:

$$
S_{1}=\frac{\Delta R^{I I}}{\Delta R^{I}} \quad S_{0}=\left(\frac{\Delta R^{I I}}{\Delta R^{I}}\right)_{K F E}
$$

Indexes I and II are related to the main directions of the polarization vectors $\hat{e} \|[100]$ and $\hat{e} \|[\overline{1} 10]$, respectively. The value of the anisotropy coefficient $S_{1}$ deduced from spectra shown in Fig. 1 is equal to 1.08.

It is noteworthy that for electrooptical BFE and carrier quantization near surface $\Delta \varepsilon=\Delta \varepsilon_{s}+\Delta \varepsilon_{b}$, and $\Delta \varepsilon_{s}$, $\Delta \varepsilon_{b}$ are changes of surface and bulk dielectric permittivity components in electric field, respectively. The iso-

tropic component $\Delta \varepsilon_{s}=\Delta \varepsilon^{I} \frac{S_{0}-S_{1}}{S_{0}-1}$.

Shown in Fig. 2a are ER spectra of $i$-Ge in the case of $\hat{e} \|[100]$ (curve 1) and surface (curve 2) as well as bulk (curve 3) components. The spectrum of the bulk component (curve 3 ) as to the form and alternation order of dominating extrema corresponds to the situation when energy bands are bent up, if going towards surface. If one deals with $E_{1}$ transitions in Ge under BFE conditions, this situation takes place only at presence of an extremum in the dependency of semiconductor electrostatic potential, $V$, on $x$ coordinate. In this case, signals of interband phototunneling formed at different sides of the extremum are opposite in their phases, and the net spectrum is determined by the phase of the dominating signal. It should be similar to that obtained by superposition of ER spectra for $n$ - and $p$-type samples measured in the range of enriching band slopes under BFE

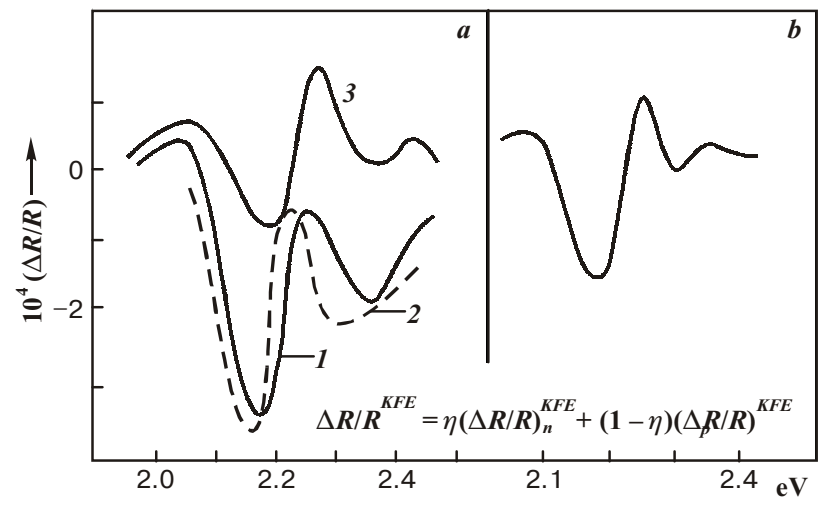

Fig. 2. $a$ - ER spectrum (1), its isotropic (2) and anisotropic (3) components obtained for the case of the intrinsic Ge etched surface. $b$ - Modelling the bulk (anisotropic) component of ER spectrum by combination of such spectra for $n$ - and $p$-types of Ge. The mixing coefficient was taken as $\eta=0.5$.

SQO, 6(2), 2003 


\section{P.A. Gentsar et al.: Effects of interband phototunneling and ...}

absence conditions and mixing coefficient $\eta=0.5$. And really, superposition of these spectra results in the curve close in its form (Fig. 2b), which can be interpreted as supplementary argument of the extremum existence.

The appearance of this extremum is obviously related with the fact that the electron wave function is equal to zero at the surface and/or with action of mirror image forces.

To estimate electric field of mirror image forces, we shall make the following. It is known that there is an intrinsic oxide layer at the surface of chemically etched semiconductor. In $\mathrm{Ge}$ case, it can be $\mathrm{GeO}_{2}$ or $\mathrm{GeO}$, i.e. dielectric substance. Let us consider the system semiconductor-dielectric-electrolyte. The charge $q$ is in semiconductor at the distance $a$ from the semiconductor-dielectric interface. The dielectric thickness is $c$. One can find values of potential and strength of mirror image forces that act in the chosen coordinate system. Direct $z$ axis along the line perpendicular to the surface. Then the positions of interfaces between substances 1 and 2, 2 and 3 will be as follows: $z=a, z=a+c$ (Fig. 3).

We shall search for the potential in the form

$$
\begin{aligned}
& \varphi_{1}=\frac{q}{\varepsilon_{1}} \int_{0}^{\infty} I_{0}\left(k r_{1}\right) e^{-k|z|} d k+\int_{0}^{\infty} A_{1}(k) I_{0}\left(k r_{1}\right) e^{k z} d k \\
& /-\infty<z<a / \\
& \varphi_{2}=\int_{0}^{\infty} B_{1}(k) I_{0}\left(k r_{1}\right) e^{-k z} d k+\int_{0}^{\infty} B_{2}(k) I_{0}\left(k r_{1}\right) e^{k z} d k
\end{aligned}
$$

$|a<z<b|$

$\varphi_{3}=\int_{0}^{\infty} A_{2}(k) I_{0}\left(k r_{1}\right) e^{-k z} d k$

$|b<z<\infty, b=a+c|$

Boundary conditions take a look [12]:

$$
\begin{aligned}
& \varphi_{1}=\left.\varphi_{2}\right|_{z=a} \\
& \varepsilon_{1} \frac{\partial \varphi_{1}}{\partial z}=\left.\varepsilon_{2} \frac{\partial \varphi_{2}}{\partial z}\right|_{z=a}
\end{aligned}
$$

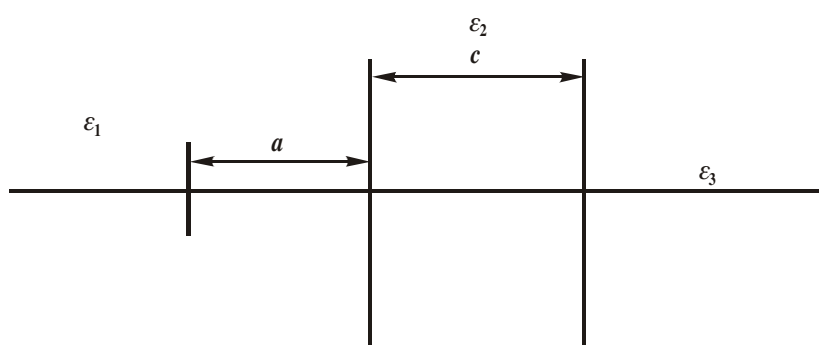

Fig. 3. Semiconductor-dielectric-electrolyte system. Dielectric permittivities of media are designated as $\varepsilon_{1}, \varepsilon_{2}$ and $\varepsilon_{3}$, respectively.

$$
\begin{aligned}
& \varphi_{2}=\left.\varphi_{3}\right|_{z=a+c=b} \\
& \varepsilon_{2} \frac{\partial \varphi_{2}}{\partial z}=\left.\varepsilon_{3} \frac{\partial \varphi_{3}}{\partial z}\right|_{z=a+c=b}
\end{aligned}
$$

Using (11) one can obtain the following system of four linear equations:

$$
\begin{aligned}
& e^{k a} A_{1}(k)-e^{-k a} B_{1}(k)-e^{k a} B_{2}(k)=-\frac{q}{\varepsilon_{1}} e^{-k a} \\
& \varepsilon_{1} e^{k a} A_{1}(k)+\varepsilon_{2} e^{-k a} B_{1}(k)-\varepsilon_{2} e^{k a} B_{2}(k)=q e^{-k a} \\
& e^{-k b} A_{2}(k)-e^{-k b} B_{1}(k)-e^{k b} B_{2}(k)=0 \\
& \varepsilon_{3} e^{-k b} A_{2}(k)-\varepsilon_{2} e^{-k b} B_{1}(k)+\varepsilon_{2} e^{k b} B_{2}(k)=0
\end{aligned}
$$

with four unknowns $A_{1}(k), A_{2}(k), B_{1}(k)$ and $B_{2}(k)$.

The system (12) determinant and those of above unknowns are as follows

$$
\begin{aligned}
& \Delta_{\mathrm{A}_{1}(k)}=\frac{q}{\varepsilon_{1}}\left\{\left(\varepsilon_{2}-\varepsilon_{1}\right)\left(\varepsilon_{2}+\varepsilon_{3}\right) e^{-2 k a}+\right. \\
& \left.+\left(\varepsilon_{1}+\varepsilon_{2}\right)\left(\varepsilon_{3}-\varepsilon_{2}\right) e^{-2 k b}\right\} \\
& \Delta_{\mathrm{A}_{2}(k)}=-4 q \varepsilon_{2} \\
& \Delta_{B_{1}(k)}=-2 q\left(\varepsilon_{2}+\varepsilon_{3}\right) \\
& \Delta_{\mathrm{B}_{2}(k)}=-2 q\left(\varepsilon_{2}+\varepsilon_{3}\right) e^{-2 k b} \\
& \Delta=-\left(\varepsilon_{1}+\varepsilon_{2}\right)\left(\varepsilon_{2}+\varepsilon_{3}\right)+\left(\varepsilon_{2}-\varepsilon_{1}\right)\left(\varepsilon_{2}-\varepsilon_{3}\right) e^{-2 k c}
\end{aligned}
$$

Then, unknowns take a look:

$$
\begin{aligned}
& A_{1}(k)=\frac{q}{\varepsilon_{1}} \frac{\tilde{\varepsilon}_{12} e^{-2 k a}+\widetilde{\varepsilon}_{23} e^{-2 k b}}{1-\widetilde{\varepsilon}_{21} \widetilde{\varepsilon}_{23} e^{-2 k c}} \\
& A_{2}(k)=\frac{\frac{4 q \varepsilon_{2}}{\left(\varepsilon_{1}+\varepsilon_{2}\right)\left(\varepsilon_{2}+\varepsilon_{3}\right)}}{1-\widetilde{\varepsilon}_{21} \tilde{\varepsilon}_{23} e^{-2 k c}} \\
& B_{1}(k)=\frac{\frac{2 q}{\left(\varepsilon_{1}+\varepsilon_{2}\right)}}{1-\widetilde{\varepsilon}_{21} \tilde{\varepsilon}_{23} e^{-2 k c}} \\
& B_{2}(k)=\frac{2 q \tilde{\varepsilon}_{23} \frac{1}{\left(\varepsilon_{1}+\varepsilon_{2}\right)} e^{-2 k b}}{1-\widetilde{\varepsilon}_{21} \widetilde{\varepsilon}_{23} e^{-2 k c}}
\end{aligned}
$$

where

$$
\begin{array}{ll}
\tilde{\varepsilon}_{12}=\frac{\varepsilon_{1}-\varepsilon_{2}}{\varepsilon_{1}+\varepsilon_{2}}=-\widetilde{\varepsilon}_{21} & \widetilde{\varepsilon}_{21}=\frac{\varepsilon_{2}-\varepsilon_{1}}{\varepsilon_{1}+\varepsilon_{2}} \\
\tilde{\varepsilon}_{23}=\frac{\varepsilon_{2}-\varepsilon_{3}}{\varepsilon_{2}+\varepsilon_{3}}=-\widetilde{\varepsilon}_{32} & \widetilde{\varepsilon}_{32}=\frac{\varepsilon_{3}-\varepsilon_{2}}{\varepsilon_{2}+\varepsilon_{3}}
\end{array}
$$


Substituting (14) into (8-10) and using known relations $[13,14]$

$$
\begin{aligned}
& \int_{0}^{\infty} e^{-k|z|} I_{0}\left(k r_{1}\right) d k=\frac{1}{\sqrt{r_{1}^{2}+z^{2}}} \\
& \int_{0}^{\infty} \frac{e^{-q x} d x}{1-a e^{-p x}}=\sum_{k=0}^{\infty} \frac{a^{n}}{q+k p}[0<a<1] \\
& r_{1}=\sqrt{x^{2}+y^{2}}=0
\end{aligned}
$$

we can find potentials of mirror image forces

$$
\begin{aligned}
& \varphi_{1}=\frac{q}{\varepsilon_{1}} \frac{1}{z}+\frac{q}{\varepsilon_{1}} \times \\
& \times\left[\widetilde{\varepsilon}_{12} \sum_{n=0}^{\infty} \frac{\left(\widetilde{\varepsilon}_{21} \cdot \widetilde{\varepsilon}_{23}\right)^{n}}{[2 a+2 n c-z]}+\widetilde{\varepsilon}_{23} \sum_{n=0}^{\infty} \frac{\left(\widetilde{\varepsilon}_{21} \cdot \widetilde{\varepsilon}_{23}\right)^{n}}{[2 a+2(n+1) c-z]}\right] \\
& \varphi_{2}=\frac{2 q}{\left(\varepsilon_{1}+\varepsilon_{2}\right)} \times \\
& \times\left\{\sum_{n=0}^{\infty} \frac{\left(\widetilde{\varepsilon}_{21} \cdot \widetilde{\varepsilon}_{23}\right)^{n}}{[2 n c+z]}+\widetilde{\varepsilon}_{23} \sum_{n=0}^{\infty} \frac{\left(\widetilde{\varepsilon}_{21} \cdot \widetilde{\varepsilon}_{23}\right)^{n}}{[2 a+2(n+1) c-z]}\right\} \\
& \varphi_{3}=\frac{4 q \varepsilon_{2}}{\left(\varepsilon_{1}+\varepsilon_{2}\right)\left(\varepsilon_{2}+\varepsilon_{3}\right)} \sum_{n=0}^{\infty} \frac{\left(\widetilde{\varepsilon}_{21} \cdot \widetilde{\varepsilon}_{23}\right)^{n}}{[2 n c+z]} .
\end{aligned}
$$

Here, we can proceed to the analysis of the potential $\varphi_{1}$ :

$$
\begin{aligned}
& \varphi_{1}=\frac{q}{\varepsilon_{1}} \frac{1}{z}+\frac{q}{\varepsilon_{1}} \times \\
& \times\left[\widetilde{\varepsilon}_{12} \sum_{n=0}^{\infty} \frac{\left(\tilde{\varepsilon}_{21} \cdot \widetilde{\varepsilon}_{23}\right)^{n}}{[2 a+2 n c-z]}+\widetilde{\varepsilon}_{23} \sum_{n=0}^{\infty} \frac{\left(\widetilde{\varepsilon}_{21} \cdot \widetilde{\varepsilon}_{23}\right)^{n}}{[2 a+2(n+1) c-z]}\right]
\end{aligned}
$$

This potential $\varphi_{1}$ consist of the point charge potential $\varphi_{0}$ and image force potential $\varphi$ :

$$
\begin{aligned}
& \varphi_{1}=\varphi_{0}+\varphi \\
& \varphi_{0}=\frac{q}{\varepsilon_{1}} \frac{1}{z} \\
& \varphi=\frac{q}{\varepsilon_{1}}\left[\tilde{\varepsilon}_{12} \sum_{n=0}^{\infty} \frac{\left(\widetilde{\varepsilon}_{21} \cdot \widetilde{\varepsilon}_{23}\right)^{n}}{[2 a+2 n c-z]}+\widetilde{\varepsilon}_{23} \sum_{n=0}^{\infty} \frac{\left(\widetilde{\varepsilon}_{21} \cdot \widetilde{\varepsilon}_{23}\right)^{n}}{[2 a+2(n+1) c-z]}\right]
\end{aligned}
$$

If our observation point is in the charge position, then the potential of mirror image forces $\varphi$ takes a look

$$
\varphi=\frac{q}{\varepsilon_{1}}\left\{\widetilde{\varepsilon}_{12} \sum_{n=0}^{\infty} \frac{\left(\widetilde{\varepsilon}_{21} \cdot \widetilde{\varepsilon}_{23}\right)^{n}}{[2 a+2 n c]}+\widetilde{\varepsilon}_{23} \sum_{n=0}^{\infty} \frac{\left(\widetilde{\varepsilon}_{21} \cdot \widetilde{\varepsilon}_{23}\right)^{n}}{[2 a+2(n+1) c]}\right\}
$$

When $c \rightarrow \infty, \varphi$ is equal

$\left.\varphi\right|_{c \rightarrow \infty}=\frac{q}{2 a \varepsilon_{1}} \widetilde{\varepsilon}_{12}$

In [12], the force acting on a charge (image force) in this system is equal

$$
F=\left(\frac{e}{2 h}\right)^{2} \frac{\varepsilon_{1}-\varepsilon_{2}}{\varepsilon_{1}\left(\varepsilon_{1}+\varepsilon_{2}\right)}
$$

The respective potential can be expressed as

$$
\varphi=\int_{0}^{2 h} \frac{F}{e} d r=\left.\frac{e}{(2 h)^{2} \varepsilon_{1}} \widetilde{\varepsilon}_{12} \cdot r\right|_{0} ^{2 h}=\frac{e}{2 h \varepsilon_{1}} \widetilde{\varepsilon}_{12}
$$

The coincidence of (21) and (23) is indicative of our estimation validity.

When oxide is absent and $c=0$, then

$$
\begin{aligned}
& \left.\varphi\right|_{c=0}=\frac{q}{\varepsilon_{1}}\left[\widetilde{\varepsilon}_{12} \sum_{n=0}^{\infty} \frac{\left(\widetilde{\varepsilon}_{21} \cdot \widetilde{\varepsilon}_{23}\right)^{n}}{2 a}+\right. \\
& \left.+\widetilde{\varepsilon}_{23} \sum_{n=0}^{\infty} \frac{\left(\widetilde{\varepsilon}_{21} \cdot \widetilde{\varepsilon}_{23}\right)^{n}}{2 a}\right]=\frac{q}{2 \varepsilon_{1} a} \times
\end{aligned}
$$

$$
\begin{aligned}
& \times\left\{\sum_{n=0}^{\infty}\left(\widetilde{\varepsilon}_{12}+\widetilde{\varepsilon}_{23}\right)\left(\widetilde{\varepsilon}_{21} \cdot \widetilde{\varepsilon}_{23}\right)^{n}\right\}= \\
& =\frac{q}{2 \varepsilon_{1} a} \frac{\widetilde{\varepsilon}_{12}+\widetilde{\varepsilon}_{23}}{1-\widetilde{\varepsilon}_{21} \cdot \widetilde{\varepsilon}_{23}}=\frac{q}{2 \varepsilon_{1} a} \widetilde{\varepsilon}_{13} .
\end{aligned}
$$

Let us find the electric field of mirror image forces:

$\vec{E}=-\operatorname{grad} \varphi$

Substituting the formula (19) into (25) one can get

$$
\begin{aligned}
& E=-\frac{q}{\varepsilon_{1}}\left\{\widetilde{\varepsilon}_{12} \sum_{n=0}^{\infty} \frac{\left(\widetilde{\varepsilon}_{21} \cdot \widetilde{\varepsilon}_{23}\right)^{n}}{[2 a+2 n c-z]^{2}}+\right. \\
& \left.+\widetilde{\varepsilon}_{23} \sum_{n=0}^{\infty} \frac{\left(\widetilde{\varepsilon}_{21} \cdot \widetilde{\varepsilon}_{23}\right)^{n}}{[2 a+2(n+1) c-z]^{2}}\right\}
\end{aligned}
$$

In the point of observation, the electric field of image forces takes the following form: 


\section{P.A. Gentsar et al.: Effects of interband phototunneling and ...}

$$
\begin{aligned}
& E=-\frac{q}{\varepsilon_{1}}\left\{\widetilde{\varepsilon}_{12} \sum_{n=0}^{\infty} \frac{\left(\widetilde{\varepsilon}_{21} \cdot \widetilde{\varepsilon}_{23}\right)^{n}}{[2 a+2 n c]^{2}}+\right. \\
& \left.\widetilde{\varepsilon}_{23} \sum_{n=0}^{\infty} \frac{\left(\widetilde{\varepsilon}_{21} \cdot \widetilde{\varepsilon}_{23}\right)^{n}}{[2 a+2(n+1) c]^{2}}\right\}
\end{aligned}
$$

Thus, we have the potential and field of mirror image forces:

$$
\begin{aligned}
& \varphi=\frac{q}{2 \varepsilon_{1}}\left\{\widetilde{\varepsilon}_{12} \sum_{n=0}^{\infty} \frac{\left(\widetilde{\varepsilon}_{21} \cdot \widetilde{\varepsilon}_{23}\right)^{n}}{[a+c n]}+\right. \\
& \left.+\widetilde{\varepsilon}_{23} \sum_{n=0}^{\infty} \frac{\left(\widetilde{\varepsilon}_{21} \cdot \widetilde{\varepsilon}_{23}\right)^{n}}{[a+c(n+1)]}\right\} \\
& E=-\frac{q}{4 \varepsilon_{1}}\left\{\widetilde{\varepsilon}_{12} \sum_{n=0}^{\infty} \frac{\left(\widetilde{\varepsilon}_{21} \cdot \widetilde{\varepsilon}_{23}\right)^{n}}{[a+c n]^{2}}+\right. \\
& \left.+\widetilde{\varepsilon}_{23} \sum_{n=0}^{\infty} \frac{\left(\widetilde{\varepsilon}_{21} \cdot \widetilde{\varepsilon}_{23}\right)^{n}}{[a+c(n+1)]^{2}}\right\}
\end{aligned}
$$

Now we can get numerical estimations of these values. Let us transform formulae (28) and (29) in the following manner:

$$
\begin{aligned}
& \varphi=\frac{q}{2 \varepsilon_{1}}\left\{\widetilde{\varepsilon}_{12} \sum_{n=0}^{\infty} \frac{\left(\widetilde{\varepsilon}_{12} \cdot \widetilde{\varepsilon}_{32}\right)^{n}}{[a+c n]}-\right. \\
& \left.-\widetilde{\varepsilon}_{32} \sum_{n=0}^{\infty} \frac{\left(\widetilde{\varepsilon}_{12} \cdot \widetilde{\varepsilon}_{32}\right)^{n}}{[a+c(n+1)]}\right\} \\
& E=-\frac{q}{4 \varepsilon_{1}}\left\{\widetilde{\varepsilon}_{12} \sum_{n=0}^{\infty} \frac{\left(\widetilde{\varepsilon}_{12} \cdot \widetilde{\varepsilon}_{32}\right)^{n}}{[a+c n]^{2}}-\right. \\
& \left.-\widetilde{\varepsilon}_{32} \sum_{n=0}^{\infty} \frac{\left(\widetilde{\varepsilon}_{12} \cdot \widetilde{\varepsilon}_{32}\right)^{n}}{[a+c(n+1)]^{2}}\right\}
\end{aligned}
$$

To make there numerical calculations more convenient, one can write (30) and (31) in the form:

$$
\begin{aligned}
& \varphi=\frac{q}{2 \varepsilon_{1} a}\left\{\widetilde{\varepsilon}_{12} \sum_{n=0}^{\infty} \frac{\left(\widetilde{\varepsilon}_{12} \cdot \widetilde{\varepsilon}_{32}\right)^{n}}{\left[1+\frac{c}{a} n\right]}-\right. \\
& \left.-\widetilde{\varepsilon}_{32} \sum_{n=0}^{\infty} \frac{\left(\widetilde{\varepsilon}_{12} \cdot \widetilde{\varepsilon}_{32}\right)^{n}}{\left[1+\frac{c}{a}(n+1)\right]}\right\}
\end{aligned}
$$

$$
\begin{aligned}
& E=-\frac{q}{4 \varepsilon_{1} a^{2}}\left\{\widetilde{\varepsilon}_{12} \sum_{n=0}^{\infty} \frac{\left(\widetilde{\varepsilon}_{12} \cdot \widetilde{\varepsilon}_{32}\right)^{n}}{\left[1+\frac{c}{a} n\right]^{2}}-\right. \\
& \left.-\widetilde{\varepsilon}_{32} \sum_{n=0}^{\infty} \frac{\left(\widetilde{\varepsilon}_{12} \cdot \widetilde{\varepsilon}_{32}\right)^{n}}{\left[1+\frac{c}{a}(n+1)\right]^{2}}\right\}
\end{aligned}
$$

Fig. 4 shows the dependence of the sought potential $\varphi$ and electric field $E$ on the parameter $a$ under the following values of material constauts $[15,16]$ :

$\varepsilon_{1}=16$ (for $G e$ )

$\varepsilon_{2}=\frac{\left(n_{\mathrm{GeO}}\right)^{2}+\left(n_{\mathrm{GeO}}\right)^{2}}{2}=2.65$

$\varepsilon_{3}=(1.35)^{2}=1.82$

$c=15 \AA$.

Thus, the results obtained can be formulated as follows:

for etched surface of intrinsic germanium $(i-\mathrm{Ge})$, the spectrum of the bulk ER component, as to its shape and order of alternation of dominating structures, corresponds to the situation when energy bands are bent up in the direction to this surface. If taking info accounts the effect of filling the bands, this situation can take place only at presence of some extremum in the energy diagram of semiconductor. Appearance of such extremum can be ascribed to zero value of the electron wave function at the surface or some effect of mirror image forces.
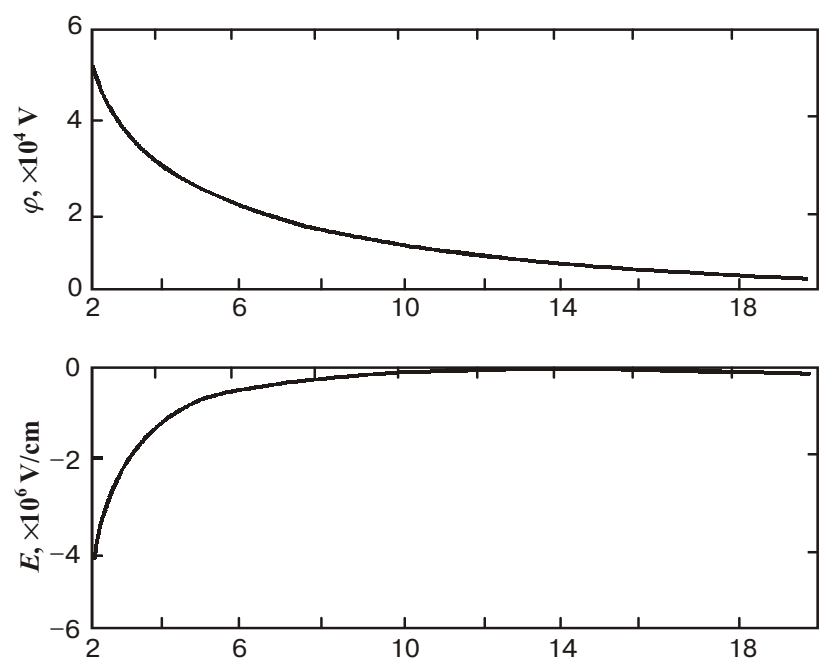

Fig. 4. Dependencies of the potential $\varphi$ and field strength $E$ of mirror image electric field on the parameter $a$. 


\section{References}

1. V.A. Tyagai, O.V. Snitko. Light electroreflection in semiconductors. Naukova dumka, 1980, 302p, (in Russian).

2. R.V. Kuz'menko, A.V. Ganzha, E.P. Domashevskaya, V. Kirher, Sh. Hildebrandt. Generalized multiplayer model for the quantitative analysis of electromodulation components in electroreflectance spectra and photoreflection of semiconductors in the range of the fundamental transition $E_{0} / /$ Semiconductors (St.-Petersburg) 34 (9), p.1086-1092 (2000).

3. R.V. Kuz'menko, A.V. Ganzha, E.P. Domashevskaya. Combined method of studying multi-component photoreflectance spectra in semiconductors // Semiconductors (St.-Petersburg) 36(1), p. $52-58$ (2002).

4. N. Bottka and D.L. Johnson, Theory of band population effects in electroreflectance // Phys. Rev. B-Solid State, 11(8), p. 2969-2978 (1975).

5. R. del Sole, Surface effects in electroreflectance // Solid State Commun. 19(2), p.207-211 (1976).

6. L.P. Abakyants, P.Ya. Bokov, G.B. Galliev, V.A. Kaminsky, V.A. Kul'bachinsky, V.G. Mokerov, A.V. Chervyakov. Investifation of quantum size effects in bound quantum wells $\mathrm{Al}_{\mathrm{x}} \mathrm{Ga}_{1-\mathrm{x}} \mathrm{As} / \mathrm{GaAs} / \mathrm{Al}_{\mathrm{x}} \mathrm{Ga}_{1-\mathrm{x}} \mathrm{As}$ using the method of photoreflection spectroscopy // Optika i spektroskopiya 93(6), p. 929-934 (2002), in Russian.

7. R. del Sole and D.E. Aspnes, Effect of surface and nonuniform field in electroreflectance: Application to Ge // Phys. Rev. B17(8), p. 3310-3317 (1978).

8. A.M. Evstigneyev, O.V. Snitko, L.B. Artamonov, P.A. Gentsar, A.N. Krasiko, Surface contribution into the electroreflection effect and its separation at presence of polarization anisotropy // Ukrainskii fizicheskii zhurnal 31(5), p. 756-759 (1986), in Russian.

9. A.M. Evstigneyev, O.V. Snitko, A.N. Krasiko, P.A. Gentsar, E.V. Mozdor, Study of mechanically disturbed Ge surface using the method of anisitropic electroreflectance // ibid. 32(2), p. 269-272 (1987), in Russian.

10. A.O. Volkov and O.A. Ryabushkin, Microwave-optical modulation spectroscopy for studying semiconductor structures // Pribory i tekhnika eksperimenta 5, p. 121-125, in Russian.

11. P.A. Gentsar, Effects of interband phototunneling and filling the bands in Ge electroreflection spectra. In: Abstracts of «Republic Conference of Young Scientists for Actual Problems of Physics», Dilizhan, 14-18 October, 1985. Edited by the Academy of Sciences of Armenian SSR. Erevan, 1985, p. 249.

12. L.D. Landau and E.M. Livshits, Electrodynamics of continuous media, V.8, Nauka, Moscow, 1982, 624p. (in Russian).

13. A.N. Tikhonov and A.A. Samarsky. Equations of mathematical physics, Nauka, Moscow, 1977, 736p. (in Russian).

14. I.S. Gradshtein and I.M. Ryzhik. Tables of integrals, sums, sets and products, Gos. izd. fiz.-mat. literatury, Moscow, 1963, 1100 p. (in Russian).

15. Handbook for chemists. The main proportions of non-organic and organic compounds, V.2. Gos. nauchno-teknicheskoye izd-vo chim. literatury, Leningrad-Moscow, 1951, 1148p. (in Russian).

16. Handbook for chemists. Chemical equilibriun and kinetics of solution properties. Electrode processes. Khimiya, MoscowLeningrad, 1951, 1004 p. (in Russian). 